\title{
Density Functional Theory Analysis of Carboxylate-Bridged Diiron Units in Two-Dimensional Metal-Organic Grids
}

\author{
Ari P. Seitsonen, ${ }^{*} \uparrow$ Magali Lingenfelder, $\neq$ Hannes Spillmann, ${ }^{\ddagger}$ Alexandre Dmitriev, ${ }^{\ddagger}$ \\ Sebastian Stepanow, ${ }^{\ddagger}$ Nian Lin, ${ }^{\ddagger}$ Klaus Kern,,${ }^{\ddagger}$, and Johannes V. Barth ${ }^{\star},, \mathbb{\pi}$ \\ IMPMC, CNRS \& Université Pierre et Marie Curie, 4 place Jussieu, case 115, F-75252 Paris, France, \\ Max-Planck-Institut für Festkörperforschung, Heisenbergstr. 1, D-70569 Stuttgart, Germany, \\ Institut de Physique des Nanostructures, EPF Lausanne, CH-1015 Lausanne, Switzerland, and \\ Departments of Chemistry and Physics \& Astronomy, UBC Vancouver, BC V6T 1Z4, Canada
}

Received January 10, 2006; E-mail: ari.p.seitsonen@iki.fi; jvb@chem.ubc.ca

Supramolecular engineering of metal-organic architectures on well-defined substrates is currently emerging as a promising route toward novel nanoscale systems and functional surfaces. ${ }^{1}$ Investigations in this field, relying on scanning tunneling microscopy (STM) observations under ultrahigh vacuum conditions, revealed that on atomically clean metal surfaces an intriguing variety of lowdimensional metallosupramolecular arrangements can be synthesized by controlled deposition of polytopic linker molecules and transition metal centers. In particular, the assembly of nanoporous $\mathrm{Fe}$-carboxylate metal-organic coordination networks (MOCNs) was achieved. ${ }^{2}$ Such 2-D MOCNs comprise regular arrays of coordinatively unsaturated metal centers and distinct nanocavities which proved to be suitable as versatile and robust templates for the organization of appropriate guest species. However, while the STM investigations allow for molecular-level elucidation of morphology, dynamics, and host-guest chemistry of low-dimensional metal-organic grids, there is a clear need to rationalize the corresponding atomic structure, electronic properties, and chemical bonding in depth. Here we report an ab initio theoretical analysis for an exemplary MOCN, which was modeled using density functional theory (DFT) calculations. We notably address the diiron center key feature encountered in a series of fully 2-D reticulated $\mathrm{Fe}$-carboxylate networks, which is reminiscent of catalytically active carboxylate-bridged diiron sites in metalloproteins. ${ }^{4}$ Specifically, we modeled the nanoporous $\mathrm{Fe}$-diterephthalate array fabricated from Fe atoms and 1,4-benzenedicarboxylic acid (terephthalic acid, tpa) molecules on $\mathrm{Cu}(100){ }^{3}$

The constant current STM image depicted in Figure 1A shows the $\alpha$-isomer of the 2-D Fe-terephthalate MOCN with its distinct nanocavities. On the basis of the topographic data, the schematic model superimposing the image was derived, suggesting diiron centers connected by both unidentate and chelating bidentate bonds to the adjacent diterephthalate linkers. ${ }^{2,3}$ The STM observations clearly indicate a $(6 \times 4)$ metal-organic array fully commensurate with the underlying copper square lattice, where the protrusions associated with the closest $\mathrm{Fe}$ atoms are found at a distance corresponding to roughly two $\mathrm{Cu}(100)$ lattice units $(a=2.55 \AA)$. These structural features were employed as a starting point for the present DFT study, where we assumed that the Fe centers reside near the energetically favorable 4-fold hollow sites.

The calculations were performed with the Generalized Gradient Approximation as the exchange-correlation functional of the KohnSham equations in the code VASP. ${ }^{5}$ The electronic wave functions were expanded in a plane wave basis set up to a cutoff energy of

† IMPMC, CNRS \& Université Pierre et Marie Curie.

¥Max-Planck-Institut für Festkörperforschung.

$\S$ EPF Lausanne.

II UBC Vancouver.

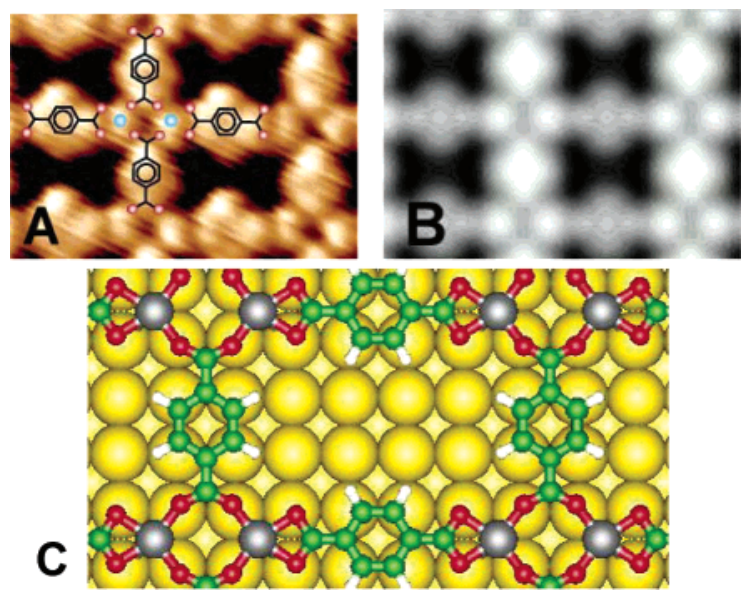

Figure 1. Fully reticulated nanoporous $\mathrm{Fe}-$ diterephthalate grid assembled on a $\mathrm{Cu}(100)$ substrate. (A) Constant current mode scanning tunneling microscopy image showing the $\alpha$-isomer comprising a $(6 \times 4)$ unit cell (image size $40 \times 30 \AA^{2}$ ). The arrangement of the tpa backbone indicates that a given molecule is engaged in either two bidentate or four unidentate carboxylate bonds to the diiron centers (marked as two blue spheres). (B) STM image simulation showing contours of constant LDOS at the sample Fermi level derived from the DFT model of the optimized structural arrangement depicted in model (C).

$37 \mathrm{Ry}$, and the core-valence interaction was modeled with the projected augmented wave method. Three substrate layers (first fully relaxed with adsorbate layer, second only laterally) together with almost $20 \AA$ of vacuum between the two surfaces of the slab were employed, and a $(2 \times 2)$ grid of Monkhorst-Pack $k$-points in the first Brillouin zone was used to approximate the integration over the reciprocal space. A top view of the fully relaxed MOCN geometry is reproduced in Figure 1C. The corresponding STM image simulation (approximated as contours of constant electron density in a small energy window, specified by two Fermi functions with a width of $0.1 \mathrm{eV}$, around the Fermi energy) agrees well with the details of the experimental finding. Notably the shape of the nanocavities and the features associated with the Fe centers and the tpa backbone are nicely reproduced. This match corroborates the original interpretation of the STM results. ${ }^{3}$ Moreover it agrees with earlier findings reporting that carboxylate groups tend to couple to the top sites of copper substrates. ${ }^{6}$

A close-up view of the carboxylate diiron coupling motif is reproduced in Figure 2. The different bonding is reflected in the opening angle defined by the carboxylate oxygen, which amounts to 124.5 and $117.3^{\circ}$ for bridging equatorial and chelating axial bonds, respectively, whereby the molecular backbone is slightly bent and residing above the plane defined by the $\mathrm{Fe}$ atoms. 


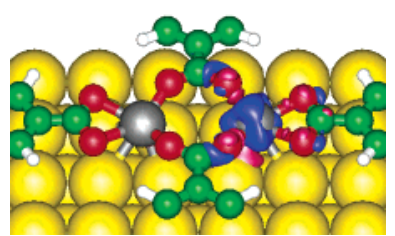

Figure 2. Perspective view of the diiron unit. The Fe charge rearrangement contour levels indicated on the right, drawn with respect to a removed iron atom, are $\pm 0.004 \mathrm{e}^{-} / \AA^{3}$, whereby the colors indicate increased (purple) and decreased (blue) electron density, respectively. Carboxylate moieties and $\mathrm{Fe}$ centers lie almost in the same plane $(\Delta z \approx 0.2 \AA)$, with the latter being displaced away from the 4-fold hollow sites, resulting in a lateral $\mathrm{Fe}-\mathrm{Fe}$ spacing of $4.4 \AA$.

Furthermore, under the influence of the carboxylate matrix, the $\mathrm{Fe}$ atoms are laterally displaced from the ideal high-symmetry substrate positions, which accounts for an $\mathrm{Fe}-\mathrm{Fe}$ spacing of $4.4 \AA$. The pertaining $\mathrm{Fe}-\mathrm{O}$ bonding distances are 2.01 (equatorial) and 2.24 (axial) $\AA$, respectively, and match with typical values encountered in 3-D Fe-carboxylates. ${ }^{7}$ For comparison, the corresponding bond lengths for a free-standing 2-D $(6 \times 4) \mathrm{Fe}-$ tpa layer were calculated as 1.92 and $2.11 \AA$, respectively. This signals that the free metalorganic array's properties are close those of the 2-D grid assembled on the surface, and that the $\mathrm{Cu}(100)$ square atomic lattice represents an excellent template. A further indication of the strong lateral $\mathrm{Fe}-$ carboxylate coupling is the fact that the $\mathrm{Fe}$-substrate bonding distance changes dramatically upon the embedding of the Fe centers in the metal-organic array; that is, compared to an isolated $\mathrm{Fe}$ adatom in a 4-fold hollow position, those in the $\mathrm{Fe}$-tpa grid are vertically lifted by $0.6 \AA$.

The electronic structure of the Fe centers was analyzed by calculating the projected density of states (DOS). The total electronic DOS of the $\mathrm{Fe}$-tpa grid projected on the $\mathrm{Cu}$ atoms in closest proximity to the Fe centers in the metal-organic grid reveals the prominent structure of the metal $d$ states setting in at an energy of $1.2 \mathrm{eV}$ below the system Fermi level $\left(E_{\mathrm{F}}\right)$. The strong hybridization between the $\mathrm{Fe}$ and $\mathrm{Cu}$ states prevents a conclusive analysis of the Fe oxidation state. The magnetic properties of the diiron centers become apparent when the DOS is projected on the Fe atomic $d$ states, as depicted in Figure 3. There is a marked splitting between the majority spin up and the minority spin down states. The latter are largely unoccupied, and the resulting spin polarization accounts for strongly magnetized Fe centers bearing a magnetic moment of $3.4 \mu_{\mathrm{B}}$, coming close to the $3.2 \mu_{\mathrm{B}}$ extracted in a recent study for $1-\mathrm{D}$ mononuclear $\mathrm{Fe}$-trimesate chains grown on the anisotropic $\mathrm{Cu}(110)$ substrate. ${ }^{8}$ Moreover, the DFT results reveal a magnetic coupling between the Fe centers. This is in line with the previously observed magnetic coupling in 3-D oxygen-bridged dinuclear iron complexes. ${ }^{9}$ Within the DFT modeling of the present

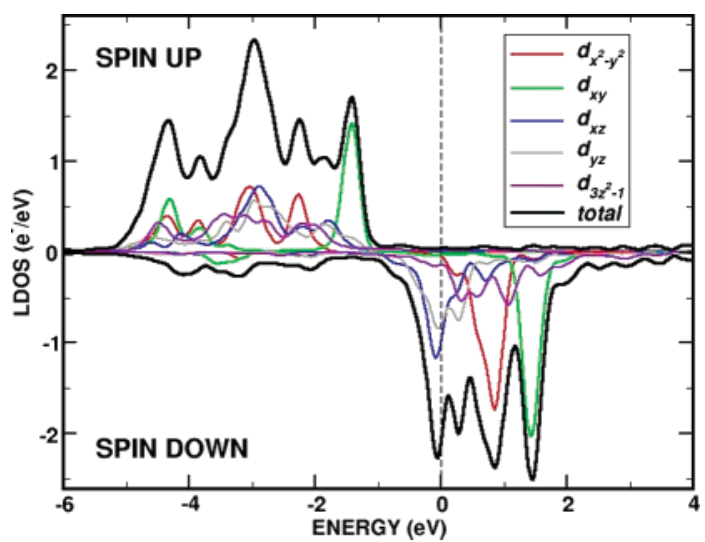

Figure 3. Spin-polarization of Fe centers as evidenced in the projected density of electronic states on the Fe atomic $d$ states.

system, ferromagnetic and antiferromagnetic coupling turned out to be very close in energy, whence no clear preference can be postulated. However, we anticipate to experimentally address this question by magnetic dichroism investigations.

In conclusion, we presented an in-depth theoretical investigation of an exemplary 2-D metal-organic coordination network comprising functional carboxylate-bridged diiron units. The achieved atomistic understanding is decisive for the considerate use of such networks as templates, guest systems, or catalysts.

Supporting Information Available: Coordinates of the network structure. This material is available free of charge via the Internet at http://pubs.acs.org.

\section{References}

(1) (a) Barth, J. V.; Costantini, G.; Kern, K. Nature 2005, 437, 671. (b) Ruben, M. Angew. Chem., Int. Ed. 2005, 44, 1594

(2) (a) Dmitriev, A.; Spillmann, H.; Lin, N.; Barth, J. V.; Kern, K. Angew. Chem., Int. Ed. 2003, 42, 2670. (b) Stepanow, S.; Lingenfelder, M.; Dmitriev, A.; Spillmann, H.; Delvigne, E.; Lin, N.; Deng, X.; Cai, C.; Barth, J. V.; Kern, K. Nat. Mater. 2004, 3, 229. (c) Clair, S.; Pons, S.; Brune, H.; Kern, K.; Barth, J. V. Angew. Chem., Int. Ed. 2005, 44, 7294 (d) Clair, S.; Pons, S.; Fabris, S.; Baroni, S.; Brune, H.; Kern, K.; Barth, J. V. J. Phys. Chem. B 2006, 110, 5627.

(3) Lingenfelder, M. A.; Spillmann, H.; Dmitriev, A.; Stepanov, S.; Lin, N.; Barth, J. V.; Kern, K. Chem.-Eur. J. 2004, 10, 1913.

(4) Merkx, M.; Kopp, D. A.; Sazinsky, M. H.; Blazyk, J. L.; Müller, J.; Lippard, S. J. Angew. Chem., Int. Ed. 2001, 40, 2782.

(5) Kresse, G.; Furthmüller, J. J. Comput. Mater. Sci. 1996, 6, 15.

(6) (a) Barlow, S. M.; Raval, R. Surf. Sci. Rep. 2003, 50, 201-341. (b) Barbosa, L. A. M. M.; Sautet, P. J. Am. Chem. Soc. 2001, 123, 6639.

(7) King, R. B., Ed. Encyclopedia of Inorganic Chemistry; Wiley: Chichester, UK, 1994.

(8) Classen, T.; Fratesi, G.; Costantini, G.; Fabris, S.; Stadler, F. L.; Kim, C.; Gironcoli, S. d.; Baroni, S.; Kern, K. Angew. Chem., Int. Ed. 2005 44,6142 .

(9) Peng, G.; Elp, J. v.; Jang, H.; Que, L.; Armstrong, W. H.; Cramer, S. P. J. Am. Chem. Soc. 1995, 117, 2515.

JA060180Y 